\title{
An Intelligent System for Electrical Energy Management in Buildings
}

\author{
Giuseppe Anastasi \\ Dept. of Information Engineering \\ University of Pisa, Italy \\ E-mail: giuseppe.anastasi@iet.unipi.it
}

\author{
Francesco Corucci \\ Dept. of Information Engineering \\ University of Pisa, Italy \\ E-mail: francesco.corucci@gmail.com
}

\author{
Francesco Marcelloni \\ Dept. of Information Engineering \\ University of Pisa, Italy \\ E-mail: francesco.marcelloni@iet.unipi.it
}

\begin{abstract}
Recent studies have highlighted that a significant part of the electrical energy consumption in residential and business buildings is due to an improper use of the electrical appliances. In this context, an automated power management system - capable of reducing energy wastes while preserving the perceived comfort level - would be extremely appealing. To this aim, we propose GreenBuilding, a sensor-based intelligent system that monitors the energy consumption and automatically controls the behavior of appliances used in a building. GreenBuilding has been implemented as a prototype and has been experimented in a real household scenario. The analysis of the experimental results highlights that GreenBuilding is able to provide significant energy savings.
\end{abstract}

Keywords - building energy efficiency; WSNs; energy awareness, building management system.

\section{INTRODUCTION}

Residential and business buildings account for approximately $20 \%$ of the overall world-wide energy consumption [1], with an increasing trend over time. The major causes of energy consumption in buildings are space heating and conditioning, water heating, lighting, and the use of computers and other electronic devices [2].

A significant part of this energy consumption is due to an improper use of such appliances and devices. Just eliminating energy wastes, without lowering the level of perceived comfort, would reduce the overall energy consumption in buildings by approximately $30 \%$ [3]. One of the main sources of energy waste is represented by electrical appliances in standby mode which accounts for approximately $10 \%$ of the overall energy consumption in buildings [2].

According to a number of studies, providing appropriate feedbacks to building occupants can significantly reduce the overall energy consumption, in the order of 5-20\% [4,5]. However, only relying on people's awareness and behavior may not be an effective approach. Indeed, a recent experimental study [6] has shown that more than 30\% energy saving was achieved immediately after installing a monitoring system in a residential household, but the percentage reduced to less than $4 \%$ one month later. An automated energy management system in addition to user cooperation would be certainly a more effective solution.

In this paper we propose GreenBuilding, a system for monitoring and controlling the power consumption of each appliance in a building. GreenBuilding exploits a nework of wireless sensors and actuators to achieve two main goals: (i) making the user aware of economic (and environmental) costs caused by an improper use of electric appliances; (ii) providing automated solutions for energy efficiency through appropriate management of appliances. We implemented GreenBuilding as a prototype and deployed it in a real household scenario. The analysis of the results obtained in the experimentation confirms that a significant amount of energy is wasted due to improper use of appliances. We show that this energy waste can be eliminated (or drastically reduced) by using a simple energy conservation rule for each specific appliance, or class of appliances.

The paper is organized as follows. Section II describes the related work. Section III introduces the GreenBuilding architecture, while Section IV describes its implementation. Section V presents the results of our experimental study. Conclusions are drawn in Section VI.

\section{RELATED WORK}

Wireless sensor networks (WSNs) have been widely used in the past for environmental monitoring applications. Recently, they have also been considered for real-time and fine-grained monitoring of electricity consumption in buildings. In [7] the author describes the design and implementation of ACme, a distributed monitoring system based on a network of AC power meters - that allows users to view their individual energy consumptions through a web interface. The same AC power meters are also used in [6] where a detailed experimental study on energy consumptions in computer science department is described. The objective of the study in [6] is to achieve full and detailed visibility of energy consumption, i.e., to understand how, where, and for whom electricity is used. Since using a dedicated power meter per electrical appliance is unpractical and expensive, the authors consider several approaches to infer the energy consumption of single appliances from aggregated measurements. The same objective is also targeted in [8] and [9]. Specifically, [8] proposes two algorithms for disaggregating the circuit-level data into device-level estimates, while [9] presents a system that is able to provide a fine-grained recognition of single appliances in real time through a single electricity sensor attached to the main electrical unit. Monitoring energy consumptions in buildings is also the goal of [10], where the authors analyze the energy consumed in various buildings in a campus, and in various floors within the same building. 
All the previous papers mainly focus on energy monitoring, while our work also addresses the problem of energy conservation through on-line power management of electrical appliances.

An automated sensor-based power-management system similar to ours is proposed in [11]. Here, however, the emphasis is mainly on architectural and design issues, rather than on an experimental analysis. On the contrary, we implemented our proposed system and deployed it in a real household scenario so as to perform an accurate experimental study. An automated system for optimizing the energy consumption of the heating, ventilation and cooling (HVAC) system in homes is presented in [12]. The proposed solution relies on occupancy sensors and switches off the HVAC system when occupants are sleeping or away from home. Unlike the proposal in [12], GreenBuilding takes a general approach, i.e., it is not tailored to a specific class of electrical appliances. [13] also proposes a distributed wireless control system for building energy efficiency. The emphasis there, however, is on efficient ways for sharing information between devices.

\section{SYSTEM ARCHITECTURE AND DESIGN}

The overall system architecture is composed of two subsystems, namely the monitoring and the control subsystems. The former acquires at prefixed time intervals measures of the energy consumption of the analyzed electrical appliances and some environmental and context information (e.g., temperature, light intensity, presence of persons). The latter exploits the data collected by the monitoring system and some energy conservation strategy to control the behavior of each single appliance. In the following, we will analyze the two subsystems in detail.

\section{A. Monitoring Subsystem}

The monitoring subsystem employs a number of electricity and environmental sensors for, respectively, measuring the power consumption of each single electrical appliance in the building, and monitoring parameters such as temperature, light intensity and human presence. Data collected by both types of sensors are wirelessly communicated to a base station located in the same floor (there is at least one base station per floor) and, then, conveyed to a central server. The communication between base stations and the server typically occurs through a wired LAN. The server processes the data and provides users with real-time and/or periodic reports on energy consumption and costs. It also sends alert messages to notify specific events (e.g. a device active when it is supposed to be inactive) suggesting possible actions to save energy.

\section{B. Control Subsystem}

The control subsystem manages the behavior of each single electrical appliance, according to energy conservation rules specified by the user, so as to minimize electricity wastes in the building. The subsystem employs one actuator for each single controlled electrical appliance and a set of controllers that coordinate the actuators. Controllers are connected to a central server, through a wired LAN technology (i.e., Ethernet). The server generates commands such as "switch on" and "switch off", for each specific appliance in the building: commands are sent to the corresponding actuator through the appropriate controller.

Actually, the sensing and actuation functionalities could be integrated in the same device, i.e., the electricity sensor could also act as actuator for the associated appliance. On the other hand, several integrated wireless sensor/actuator platforms are already currently available on the market, e.g., Plogg [14] and WiSensis [15]. Adopting an electricity sensor for each appliance is expensive and often not necessary: a single sensor can be sufficient to monitor a group of devices and take decisions on the behavior of the single device. Obviously, in order to control each device autonomously, we need to have an actuator for each device. If we adopt integrated wireless sensor/actuator platforms, we are obliged to adopt sensors for each appliance. As an alternative, we could use two different infrastructures, one for monitoring and the other for control. For example, X10 products can be used for control. The main advantage of this approach is that the X10 technology is reliable and cheap. An X10 controller sends commands to X10 actuators controlling electrical appliances through Power-Line Communication (PLC).

\section{Energy Conservation Strategies}

The system architecture has been designed with the aim of allowing a flexible and efficient management of each single appliance so as to provide the potentials for implementing appropriate energy conservation strategies for each appliance (or class of appliances). In the following we provide a (non exhaustive) list of such strategies.

- User awareness. If users are aware of the energy consumption of each single appliance, they can use electrical energy in a more efficient and convenient way. To this aim, GreenBuilding provides the users with detailed and real-time information on the instantaneous energy consumption and status (i.e., on/standby/off) of single appliances, even remotely. Further, it can also send periodic reports (e.g., by e-mail) on the use of electrical energy. Finally, GreenBuilding can alert the users by sending a message to a portable device when some specific events occur (e.g., an appliance is active when it is supposed to be inactive), also suggesting possible actions to save energy.

- Reduction of stand-by consumptions. For each specific appliance, GreenBuilding allows the user to specifiy an appropriate management strategy to reduce (or also eliminate) standby wastes. Indeed, GreenBuilding can recognize when an appliance is in standby mode and take appropriate actions to execute the strategy defined by the user. For example, the system can switch off a certain 
appliance after a predefined time interval has elapsed from when it entered the standby mode.

- Scheduling of flexible tasks. The cost of electrical energy varies during the day and typically is cheaper during the night. Thus, some energy consuming tasks that do not require a user involvement can be scheduled for execution when the energy cost is lower. GreenBuilding allows the user to specify the exact time or time interval (e.g., the slot time when the energy costs are lower) when a certain task is to be executed by a specific appliance (e.g., the washing machine). The synergy between the strategies for appropriate scheduling of flexible tasks and reduction of standby consumption can actually minimize the energy consumption of electrical appliances. For example, the washing machine can be switched on by the system only at the pre-programmed time. Once the task has been completed the appliance enters the standby mode. This is detected by the system and the washing machine is then switched off.

- Adaptive control of electrical appliances. A significant fraction of energy is wasted due to electrical appliances that are unnecessarily active, e.g., lamps switched on when the external light intensity is high, air conditioning system providing a too low ambient temperature, and so on. To eliminate this kind of wastes, GreenBuilding relies on environmental sensors capable of monitoring ambient conditions (e.g., temperature, light intensity, humidity, presence of persons, etc.). Data acquired by environmental sensors are conveyed to the central server, through base stations located at each floor, and used by the system to adapt the behavior of each single appliance, based on rules specified by the user (e.g., desired light intensity).

\section{Discussion}

The system architecture described above has a number of interesting properties. First of all, it is modular and scalable, thus making it suitable also for large buildings. Second, the system installation is non-invasive, thanks to the use of wireless sensors (and PLC devices, if present, that relies on the pre-existing electrical network). Finally, the additional energy consumed by the system is negligible with respect to the overall energy consumption of the building. In large buildings the central server could be implemented by a laptop or desktop computer, whose power consumptions are roughly $30 \mathrm{~W}$ and $100 \mathrm{~W}$, respectively. In small houses the server could be implemented through a low-power device, e.g., the home router connecting to the Internet, whose power consumption is typically less than $10 \mathrm{~W}$ (moreover, the home router is typically active most of the time for Internet connectivity). The power consumptions of electrical sensors/actuators depend on the specific platform. For the WiSensis platform used in our testbed, the power consumption is approximately $2.5 \mathrm{~W}$ for base stations (there is only one base station per floor) and $0.2-0.4 \mathrm{~W}$ for sensors/actuators. Instead, the power consumption of environmental sensors is in the order of $\mathrm{mW}$. Finally, if a dedicate control infrastructure is used we need to take into account also the power consumption of the X10 controller (2.5W on average; one X10 controller per building is sufficient), and actuators (about $0.2 \mathrm{~W}$ ).

\section{PROTOTYPE IMPLEMENTATION}

We implemented a prototype of the previously described architecture. We used two different infrastructures for monitoring and control. The monitoring subsystem employs WiSensys [15] sensors and base stations. WiSensys sensors plug into a standard electrical outlet and provide a standard outlet at their turn. They send to the base station measurements of the power absorbed by the appliance, using a proprietary wireless communication protocol. Since there is only one base station in our testbed, it is connected to the central server through a direct RS232 link. In a real system the connection could be through an Ethernet link. The environmental sensor network consists of a number of light intensity sensors. We built light sensors by ourselves using the Arduino platform, which is a very popular opensource prototyping platform based on flexible, easy-to-use hardware and software [16]. Finally, the control infrastructure is made up by a number of X10 receivers and one X10 controller manufactured by Marmitek [17].

Figure 1 shows the software architecture of the system. Data acquired by electricity and environmental sensors are stored in a MySQL database at the server. The energy manager application, implemented in Java, communicates with the database and is responsible for (i) processing data on power consumption of appliances measured by electricity sensors, (ii) providing appropriate reports to the user, (iii) processing data originated by environmental sensors, (iv) accepting configuration parameters and energy management rules from the user, (v) sending appropriate commands to appliances - through the control network - depending on current environmental conditions and according to user's rules. The user can log on the system, even remotely, and access different kind of real-time and historical graphical information, through a set of tabs. Figure 2-a shows the tab presenting information on real-time power consumptions. The user can immediately recognize the contribution of each single appliance to the overall power consumption. The same tab also includes some (textual) information to make the user aware of possible energy wastes (e.g., appliances in standby mode). Figure 2-b shows another tab presenting historical information on the energy consumption of single appliances, i.e., the energy consumed in the last 15 days (top-side plot) and hour-by-hour in a specific day (bottomside plot). The former plot also highlights the overall standby consumption. The latter plot allows the user to better understand how energy consumptions are distributed during the day. This is particularly important if energy costs follow an hourly based tariff. 


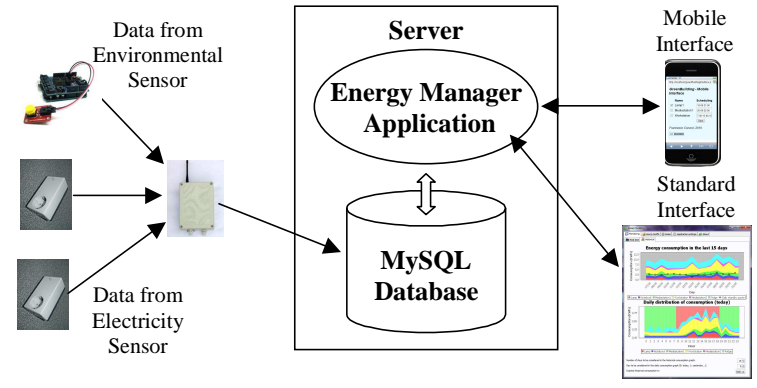

Figure 1. Software Architecture.

GreenBuilding also provides a set of tabs for introducing configuration parameters and defining energy conservation rules on single appliances or classes of appliances. Figure 3 shows how to define a rule for a certain appliance. On the left side of the tab, there is the list of all controlled appliances. Once the specific appliance has been selected, the user can specify one or more rules for (i) eliminating the standby consumption, (ii) scheduling flexible tasks at preprogrammed times, or (iii) performing appliance-specific actions (e.g., for lamps, adaptive power control based on environmental light intensity) ${ }^{1}$. With reference to standby waste, the following strategies are currently implemented in GreenBuilding.

$\mathrm{S}_{\mathrm{A}}$ : disconnect upon entering the standby mode;

$\mathrm{S}_{\mathrm{B}}$ : disconnect after a specified time interval;

$\mathrm{S}_{\mathrm{C}}$ : disconnect upon entering the standby mode if the current time is greater than a specified time threshold.

Obviously, strategy $\mathrm{S}_{\mathrm{A}}$ is the most efficient one in terms of energy conservation. However, for some kinds of appliances it may be annoying for the user. Strategy $S_{B}$ can provide a better tradeoff between energy efficiency and user satisfaction. Finally, strategy $S_{C}$ may be appropriate for appliances that are supposed to be off during certain hours, e.g., the television set during night.

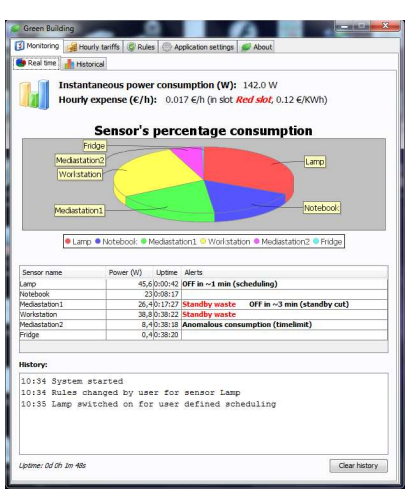

(a)

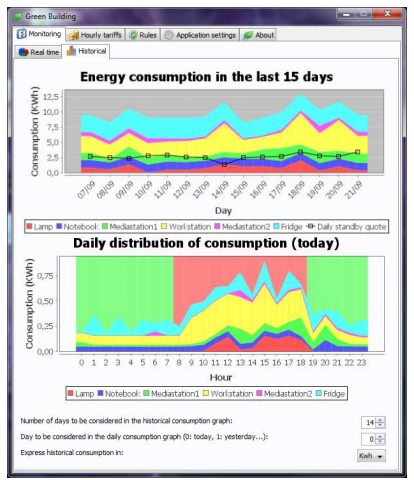

(b)
Figure 2. GreenBuilding tabs presenting: the breakdown of real time power consumptions (a), energy consumptions of different devices during a time interval and in a specific day (b).

\footnotetext{
${ }^{1}$ Depending on the specific appliance, some of the mentioned strategies
} could not be available.
In addition to the graphical interface described above, GreenBuilding also provides a simplified interface for portable devices (e.g., cell phones). The simplified interface still allows the user to look at real time and/or historical information on electrical consumptions, change configuration parameters, and specify/change rules for each single appliance (or class of appliances). In particular, the user can check the status of each appliance and switch it on and off, even remotely. Therefore, the GreenBuildingenabled mobile device can be used as a sort of remote control. In addition, the simplified interface allows the user to receive alert messages on his/her portable devices if some event occurs (e.g., an appliance that is supposed to be switched off is still in active/standby mode).

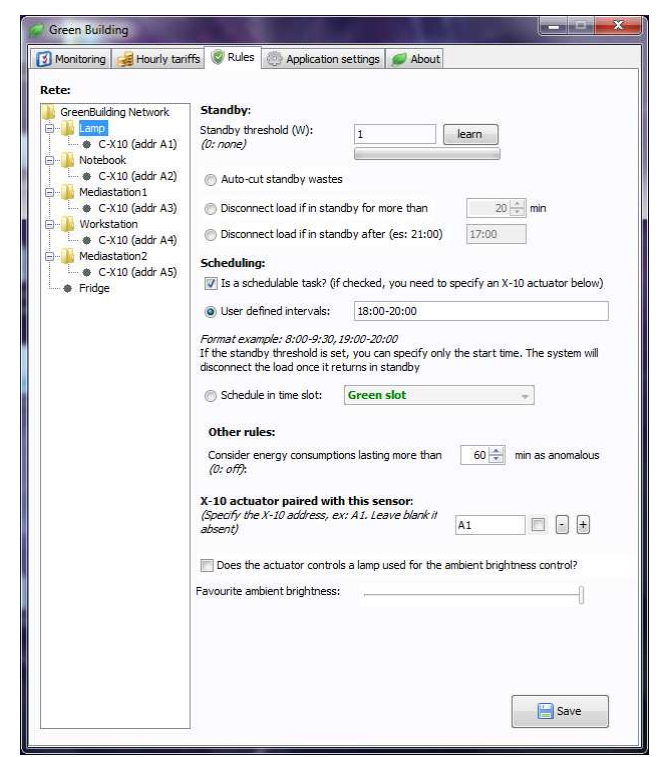

Figure 3. GreenBuilding tab for defining energy conservation rules.

\section{EXPERIMENTAL MEASUREMENTS}

To test and evaluate GreenBuilding we deployed a number of electricity and environmental sensors and X10 actuators in a real environment (i.e., a household scenario) and measured the power consumption of a set of appliances for a relatively long time interval (15 days). The objective of this experimental study is to investigate possible energy wastes and user's bad habits in using electrical appliances. We also applied some energy conservation strategies to some appliances and measured the corresponding energy savings.

\section{A. Testbed description}

We deployed electricity sensors and actuators in a household scenario, a small apartment lived by two students. Specifically, we considered the following electrical appliances.

- A room lamp consisting of three light bulbs (40W each) 
- A laptop

- A workstation composed by a PC, a router, a telephone, a printer, and a 5.1 sound system.

- A media station - throughput referred to as Media Station 1 - consisting of a CRT TV, a decoder, a VRC, a radio repeater device, and a game console.

- A second media station - throughput referred to as Media Station 2 - composed by a CRT TV, and a radio repeater device.

- A fridge

For Media Station 1, Media Station 2, and Workstation, we used a single electricity sensor to monitor the aggregate consumption of the station, while we used one X10 receiver per device composing the station. As far as environmental monitoring, we considered light sensors and monitored the light intensity in the room.

\section{B. Monitoring of daily consumption}

We measured the electrical consumptions of the above mentioned appliances for 15 days. Figure 4 shows the daily energy consumption of each considered appliance for the entire duration of the study. The plots also show the impact of standby waste, which is approximately constant over time and represents a significant fraction of the overall energy consumption. Figure 5 shows the energy consumption of each appliance in a specific day. Even if the energy consumption of each appliance varies from day to day, the general trend is similar in different days. We assume that the user is using a hourly tariff based on two time slots, i.e., a red slot (7:30am-6:30pm) when the energy cost is more expensive, and a green slot (6:30pm-7:30am) when energy is significantly cheaper. The two slots are highlighted in the plot. Data in Figure 5 show that the consumption is not distributed in a convenient way over the day since most of the energy is consumed during hours corresponding to the red slot. The energy consumption during the green slot is mainly due to standby (flat areas in the plot).

The information provided in Figure 4 and Figure 5 allow the user to better understand his/her profile in terms of electrical energy consumptions. Basically, they visually provide two important warnings to the user. He/she is not taking full advantage of the hourly tariff, and he/she is wasting a considerable amount of energy due to standby.

\section{Eliminating Standby Wastes}

Standby wastes can be highly reduced (if not eliminated) by specifying an appropriate management rule for each appliance. In the following we will assume to use the standby strategy $\mathrm{S}_{\mathrm{A}}$ for all the considered appliances, i.e., we assume that appliances are immediately switched off upon entering the standby mode. The rationale behind this choice is estimating the maximum energy saving that could be achieved by completely eliminating standby wastes. In practice, however, strategies $S_{B}$ or $S_{C}$ could be more appealing.

Figure 6 shows the overall energy consumed by the considered appliances with and without the standby waste identified by GreenBuilding. Eliminating the standby component can provide a significant energy-consumption reduction, up to $26 \%$, on average, in the considered scenario. Obviously, these results strongly depend on the specific set of appliances that has been considered in our analysis. Also, the standby waste varies significantly from one appliance to another (it is high for the workstation and media stations, while it is null for the fridge and lamps). However, even assuming a lower percentage of standby waste (e.g., 10\%), it clearly emerges that using an automated tool, like GreenBuilding, for reducing standby consumptions is convenient.

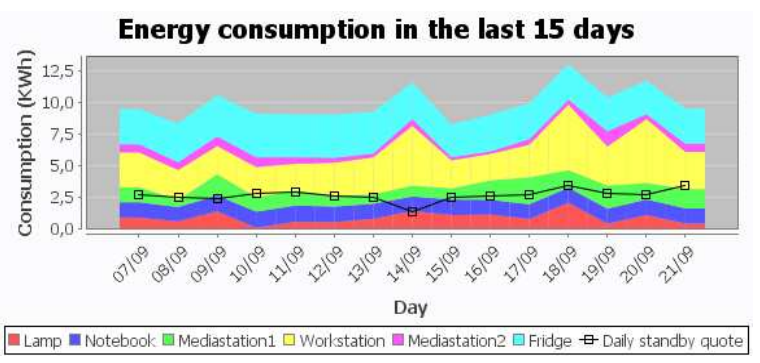

Figure 4. Daily energy consumptions and impact of standby.

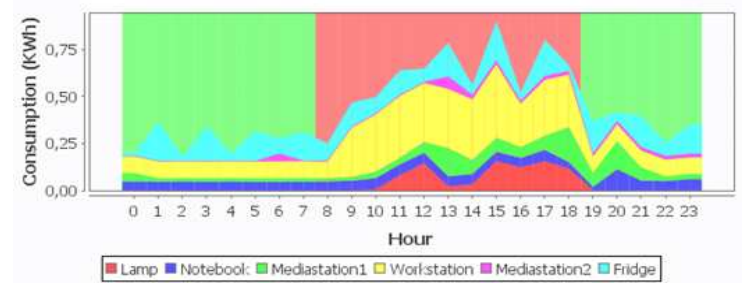

Figure 5. Energy consumptions of different appliances in a specific day.

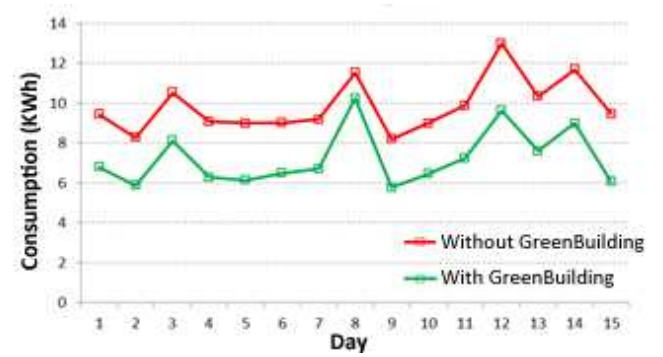

Figure 6. Energy consumption with and without GreenBuilding.

\section{Adaptive Control of Electrical Appliances}

In this section we show how GreenBuilding can save energy through adaptive control of electrical appliances. Specifically, we focused on light intensity and tried to maintain it at a constant value, by varying the power provided to the lamp, depending on the natural light intensity, so as to minimize the energy consumption. Light intensity was measured through a number of Arduino light sensors deployed in some strategic locations in the room. 
These sensors measure light intensity as a percentage ranging from $0 \%$ (no light) to $100 \%$ (very high intensity). In this use case, the user sets the desired light intensity to $50 \%$, which allowed him/her to work comfortably at the desktop or to use the workstation.

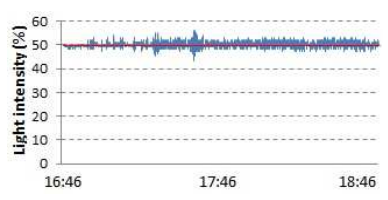

(a)

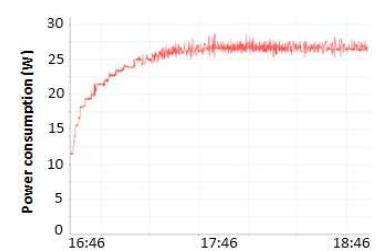

(b)
Figure 7. Light intensity in the room (a) and power consumed by the lamp (b) over time during a working session.

Figure 7 shows the average light intensity in the room as measured by light sensors ${ }^{2}$ and the corresponding power consumed by the lamp during a working session from $4: 46 \mathrm{pm}$ to $6: 46 \mathrm{pm}$ (the experiment was done in January). Figure 7-a shows that the light intensity in the room remained approximately constant around the desired value during the overall working session. Figure 7-b shows the power consumed by the lamp during the session. As expected, the power consumption tends to increase with time since the natural light intensity tends to decrease. Specifically, it gradually increases from about $11 \mathrm{~W}$ at the beginning of the test (when the sun light contributed significantly to illuminate the room) to $26 \mathrm{~W}$ at the end of the experiment (after the sunset). It may be worthwhile to emphasize that the maximum power than can be consumed by the lamp is $120 \mathrm{~W}$. The total energy consumed during the 2-hour working session was $0.05 \mathrm{KWh}$. Without adaptive control, the user would have very likely used the lamp at full power during the entire session, resulting in a total consumption of $0.24 \mathrm{KWh}$. Therefore, the use of GreenBuilding allowed saving about $79 \%$ of the energy consumed by the lamp, without reducing the level of comfort perceived by the user.

\section{CONCLUSIONS}

In this paper we have presented GreenBuilding, a system for efficient electrical-energy management in residential and business buildings. The goal of GreenBuilding is twofold. From one hand, it allows the user to better understand the energy consumption of each single appliance, thus bringing $\mathrm{him} / \mathrm{her}$ to make more intelligent choices in terms of energy consumptions. On the other hand, it allows an improved energy efficiency by means of an appropriate management of each single appliance, depending on rules specified by the user. We have implemented GreenBuilding as a prototype system and deployed it in a real household scenario. The experimental results show that GreenBuilding

\footnotetext{
${ }^{2}$ In Figure 7.a we reported the average value measured by the various light sensors in the room.
}

is able to provide significant energy savings, by eliminating standby consumptions and/or adapting the behavior of appliances to the real environmental conditions.

The main objection to using a tool like GreenBuilding is that its cost may be too high compared to the economic saving it can provide. Actually, current costs of electricity sensors and actuators are very high. However, they are expected to decrease as soon as their penetration in the market increases. In addition, next-generation electrical appliances will include built-in sensors and actuators. General Electric has already developed a complete suite of smart appliances using ZigBee [18] for wireless communication among them and/or with a central server.

\section{REFERENCES}

[1] Energy Information Administration, "International Energy Outlook 2010 - Highlights”, http://www.eia.doe.gov/oiaf/ieo/highlights.html.

[2] International Energy Agency, "Cool Appliance. Policy Strategies for Energy Efficient Homes”, Paris, France, 2003.

[3] US DOE Energy Information Administration, "Commercial Buildings Energy Consumption Survey", 2003. www.eia.doe.gov/emeu/cbecs.

[4] S. Darby, "The Effectiveness of Feedback on Energy Consumption", Techinical Report, Environmental Change Institute, University of Oxford, 2006.

[5] M. Chetty, D Tran, R. E. Grinter, "Getting to Green: Understanding Resource Consumption in the Home", Proc. UbiComp 2008, New York, USA 2008.

[6] X. Jiang, M. Van Ly, J. Taneja, P. Dutta, D. Culler, "Experiences with A High-Fidelity Wireless Building Energy Auditing Network", Proc. ACM SenSys 2009, Berkeley, USA, 2009.

[7] X. Jiang, S. Dawson-Haggerty, P. Dutta, D. Culler, "Design and Implementation of a High-Fidelity AC Metering Network", Proc. ACM/IEEE Conference on Information Processing in Sensor Networks (IPSN 2009), San Francisco, USA, 2009.

[8] A. Marchiori, Q. Han, "Using Circuit-Level Power Measurements in Household Energy Management Systems" Proceedings ACM BuildSys 2009, Berkeley, USA, 2009.

[9] A. Ruzzelli, A. Schoofs, G. O'Hare, "Real-Time Appliance Recognition And Profiling Through A Single Energy Monitoring Sensor, Proc. IEEE Conference on Sensor, Mesh and Ad Hoc Communications and Networks (SECON 2010), Boston, USA, 2010.

[10] Y. Agarwal, T. Weng, R. Gupta. "The Energy Dashboard: Improving the Visibility of eEnergy Consumption at a Campus-wide Scale", Proc. ACM BuildSys 2009, Berkeley, USA, 2009.

[11] L. Yeh, Y. Wang, Y. Tseng, "iPower: an Energy Conservation System for Intelligent Buildings by Wireless Sensor Networks", International Journal of Sensor Networks, Vol. 5, N. 1, 2009.

[12] J. Lu, T. Sookoor, G. Ge, V. Srinivasan, B. Holben, J. Stankovic, E. Field, K. Whitehouse, "The Smart Thermostat: Using Wireless Sensors to Save Energy in Homes", Proc. ACM BuildSys 2010, Zurich, CH, Nov. 2010.

[13] A. Marchiori, Q. Han, "Distributed Wireless Control for Building Energy Efficiency”, Proc. ACM BuildSys 2010, Zurich, CH, Nov. 2010.

[14] http://www.plogginternational.com/.

[15] http://www.wisensys.com/

[16] http://www.arduino.cc/.

[17] http://www.marmitek.com/en/products/home-automation-security/x10-home-automation.php.

[18] ZigBee Alliance, The ZigBee Specification version 1.0 (Q4/2007). 